Published in Progress in Organic Coatings (2006) 57(2), 91-97

\title{
The Fine Dispersion of Functionalized Carbon Nanotubes in Acrylic Latex Coatings
}

\author{
P. Vandervorst, ${ }^{*}$ C. Lei, ${ }^{*}$ Y. Lin, ${ }^{\ddagger}$ O. Dupont, ${ }^{\#}$ A.B. Dalton, ${ }^{*}$ \\ Y.-P. Sun, ${ }^{\ddagger}$ and J.L. Keddie $*^{\infty}$
}

\footnotetext{
* Department of Physics, University of Surrey, Guildford, Surrey GU2 7XH, UK

${ }^{\#}$ Cytec Surface Specialties, Anderlecht Str. 33, B-1620 Drogenbos, Belgium

${ }^{\ddagger}$ Department of Chemistry and Centre for Advanced Engineering Fibers and Films, Clemson University, Clemson, SC 29634-0973 USA
}

\begin{abstract}
Nanocomposites of a polymer and carbon nanotubes exhibit high electrical and thermal conductivity and enhanced mechanical properties in comparison to the polymer alone. Film formation from latex dispersions is an ideal way to create nanocomposite coatings with the advantages of solvent-free processing and a high uniformity of dispersion. It is shown here that carbon nanotubes functionalised with poly(vinyl alcohol) (PVA) can be blended with two types of acrylic latex to create stable colloidal dispersions without the need for added surfactant or emulsifier. Waterborne nanocomposite films with optical transparency can be formed. Microscopic analysis shows that the PVA-functionalized nanotubes are finely and uniformly dispersed in the polymer matrix.
\end{abstract}

Key words: carbon nanotubes; optical absorption; latex; nanocomposite; atomic force microscopy

\footnotetext{
${ }^{\infty}$ Corresponding author. E-mail: j.keddie@surrey.ac.uk
} 


\section{Introduction}

As a result of efforts worldwide by theorists and experimentalists, a very good understanding of the mechanisms of latex film formation has been achieved. There has been a clear identification of the factors that influence the uniformity of the drying of latex films in both directions: lateral ${ }^{1}$ (i.e. parallel to the substrate) and vertical ${ }^{2,3}$ (i.e. normal to the substrate). Furthermore, there is now an enhanced understanding of the particle deformation mechanisms because of insight gained from the process model of latex film formation, proposed by Routh and Russell. ${ }^{4}$ Experimental results are broadly consistent with the model's predictions. ${ }^{5}$

This understanding of latex film formation can now be exploited to underpin the processing of new types of coatings and adhesives. In particular, latex particles can be considered to be the "building blocks" in the creation of nanomaterials. For instance, through careful control of the film formation conditions, and through the use of core-shell ${ }^{6}$ or two-phase particles, the material's structure can be controlled on the scale of tens of nanometers. ${ }^{7}$ The blending of latex particles and inorganic nanoparticles provides a facile means of ensuring dispersion at the nanometer scale in composite coatings.

Ever since the first scientific report of carbon nanotubes (CNTs) in 1991, these materials have attracted enormous interest owing to their potential applications in field-emission devices, electronics, fibers, composites, sensors, detectors, capacitors, hydrogen storage media, and fuel cells, among others. ${ }^{8}$ As of January of 2004, 152 patents relating to nanotube applications had been issued, and anothre 274 were pending. Recent work by numerous groups has demonstrated how nanocomposites of polymers and CNTs offer the advantages of polymers, such as optical clarity, viscoelasticity and good barrier properties, combined with 
the strength ${ }^{9}$ and the high thermal and electrical conductivity ${ }^{10}$ of CNTs. Seven patents have already been issued for CNT nanocomposites and fibers. ${ }^{8}$

Four of the most common processing routes for this class of nanocomposites are (1) in-situ polymerization, ${ }^{11}(2)$ melt processing and extrusion, ${ }^{12}$ (3) casting from a common solvent, ${ }^{13}$ and (4) functionalisation of the CNT by polymers. ${ }^{14}$ Recently, nanocomposite films were prepared from CNTs that were functionalized with poly(vinyl alcohol) (PVA) to make them dispersible in water. ${ }^{15}$ The PVA-functionalized CNTs were then dispersed in an aqueous PVA solution and cast to create a nanocomposite film with a PVA matrix. This approach does not require emulsifiers or surfactants to disperse the CNTs, the final nanocomposite film is water-soluble, of course, and of limited applicability as a protective coating.

Within the past few years, it has been realised that the processing of latex dispersions offers an opportunity for blending CNTs with polymers at the nanometer scale to achieve a good dispersion in a coating matrix that is not water-soluble. The key challenge in the processing of waterborne nanocomposite coatings is to achieve the low percolation thresholds of CNTs that are predicted by theory. ${ }^{16}$ In one of the first such reports, ${ }^{17}$ a styrene-butyl acrylate latex was blended with multi-walled CNTs that had been suspended in water using sodium dodecyl sulfate (SDS). More recently, Regev et al. ${ }^{18}$ created nanocomposite films of poly(styrene) and poly(methyl methacrylate) using compression molding. They likewise did not functionalize the CNTs but dispersed them in water using SDS or gum arabic. A possible drawback of this approach is that it could increase the hydrophilicity of the coating. Grunlan et al. ${ }^{19}$ presented evidence that they had achieved a highly-uniform dispersion of single-wall carbon nanotubes (SWNTs) in a poly(vinyl acetate) latex film. The SWNTs were stabilized with either GA or poly(vinyl pyrrolidone). According to their measurements of electrical 
conductivity, percolation of the CNTs occurred at a concentration of 0.04 wt.\%. In this present work, we show that crack-free, water-resistant, and optically clear nanocomposite coatings can be created using latex processing and functionalized SWNTs. In this approach, there is no requirement for added surfactant or emulsifier.

\section{Materials and Techniques}

\subsection{Materials}

Two types of latex were used. The first type is an acrylic based on a random copolymer of 2ethyl hexyl acrylate, butyl acrylate (BA), ethyl acrylate, methyl methacrylate (MMA), and other monomers. It was prepared by semi-batch emulsion polymerization. The polymer has a low glass transition temperature $\left(T_{\mathrm{g}}=-45^{\circ} \mathrm{C}\right)$ and has applications as a pressure-sensitive adhesive. The latex dispersion has an average particle size of $190 \mathrm{~nm}$. The second latex polymer is a random copolymer of BA, MMA and methacrylic acid (MAA) in a molar ratio of BA:MMA:MAA of 85:10:5. With its $\mathrm{T}_{\mathrm{g}}$ of $20{ }^{\circ} \mathrm{C}$, this latex has applications as a protective coating. The dispersion has a solids content of $50 \mathrm{wt} . \%$ and an average particle size of $270 \mathrm{~nm}$.

SWNTs, from two different sources and with two different functionalities, were used. One type was produced by the electric arc-discharge of $\mathrm{Ni}-\mathrm{Y}$ catalyzed graphite electrodes (Carbon Solutions, Inc., California USA). The material was purified with nitric acid and left in a highly-functionalized form. The resulting SWNTs contain $4-6$ atomic $\%$ carboxylic acid $(\mathrm{COOH})$ and have a carbonaceous purity of $80-90 \%$. These $\mathrm{COOH}-\mathrm{CNT}$ s were dispersed in dimethyl formamide (DMF) with ultrasonication for $10 \mathrm{~min}$. to create a dispersion containing 0.55 wt.\% CNT. The COOH-CNT/DMF dispersion was blended with the latex dispersions while stirring, relying on the solubility of DMF and water. A second 
type of SWNT was produced via the arc-discharge method at Clemson University. The nanotubes were purified and then functionalized with poly(vinyl alcohol) (PVA) via an esterification reaction, as described elsewhere. ${ }^{15}$ A dispersion of 1.5 wt.\% PVA-CNTs in water was mixed with the concentrated latex dispersions in various proportions while stirring.

\subsection{Techniques}

With two types of latex (low- $\mathrm{T}_{\mathrm{g}}$ and high- $\mathrm{T}_{\mathrm{g}}$ ) and with two types of SWNT (COOH and PVA), nanocomposite films based on the four different combinations were created. The final concentrations of CNTs were as high as $3.5 \mathrm{wt} . \%$ on the polymer. Latex films were cast on clean, glass plates with a cube applicator having a gap of $100 \mu \mathrm{m}$. The high- $\mathrm{T}_{\mathrm{g}}$ latex films were dried at room temperature and then heated to $90{ }^{\circ} \mathrm{C}$ for $30 \mathrm{~min}$. . They typically had a final film thickness of 35 to $40 \mu \mathrm{m}$. The low- $\mathrm{T}_{\mathrm{g}}$ latex films were dried at room temperature and were typically $50 \mu \mathrm{m}$ thick.

The optical absorption coefficients of the films were obtained using a UV/visible spectrophotometer (Camspec, UK). The distribution of CNTs in the films was determined using atomic force microscopy (NT-MDT Ntegra Laboratory, Moscow, Russia) in the intermittent contact mode. High tapping free amplitudes and a relatively stiff cantilever (spring constant $k=46 \mathrm{~N} / \mathrm{m}$ ) were used. Electron microscopy was performed using an Hitachi S-4000 cold field emission scanning electron microscope using a $20 \mathrm{kV}$ beam with an emission current of $10 \mu \mathrm{A}$. Cross-sections of the high- $\mathrm{T}_{\mathrm{g}}$ latex were prepared for analysis by scanning electron microscopy (SEM), as follows. The latex dispersion were cast on sheets of poly(propylene) and film-formed in the usual way. The films were immersed in liquid nitrogen, and then the brittle films were fractured. The cross-section surfaces were coated with a thin film of $\mathrm{Au} / \mathrm{Pd}$ prior to SEM analysis. 


\section{Results}

\subsection{Colloidal Stability}

Each of the two types of CNT could be blended with each of the two types of latex dispersion. The latex dispersions containing PVA-CNTs were found to be highly stable. There is no significant amount of phase separation or sedimentation over a period of weeks. Similarly, the COOH-CNTs exhibited good stability in the low- $\mathrm{T}_{\mathrm{g}}$ latex. However, in blends with the high- $\mathrm{T}_{\mathrm{g}}$ latex, the $\mathrm{COOH}-\mathrm{CNT}$ s were seen to rise to the top of the dispersion slowly over a period of several weeks. The differences in the stability of the two types of blends might be because the low $\mathrm{T}_{\mathrm{g}}$ latex exhibits some adhesion with the COOH-CNTs that is not found with the high $\mathrm{T}_{\mathrm{g}}$ latex.

The colloidal interactions between the latex particles and the CNTs were further explored using atomic force microscopy. A very dilute blend of the low- $\mathrm{T}_{\mathrm{g}}$ latex and COOH-CNTs was cast onto freshly-cleaved mica sheets. Figure 1 shows the observed distribution of CNTs and latex particles. Nanotube structures with average lengths of $c a .1 \mu \mathrm{m}$ and heights of $c a .2$ nm (typical for a single SWNT) are observed. The resolution of the technique, as determined by the AFM tip geometry, does not enable a reliable measurement of the diameter of the structure, however. The value of the height is consistent with what is expected if exfoliation of the SWNTs had been achieved in the dispersion.

There is also evidence for attraction between the two phases, as some latex particles can be observed in Figure 1 to cluster around a SWNT. In most instances, one or more latex particle was attached to the SWNTs. Further indication of the clustering of latex particles around the CNTs was given by the visual appearance of the dispersions. When the low- $\mathrm{T}_{\mathrm{g}}$ latex was blended with the CNTs, the dispersion became blue. Although the reasons for this 
observation are not known with certainty, it is likely to be the result of the diffraction of light from the latex particles. The center-to-center distance of the particles in the clusters is on the order of one-half the wavelength of blue light. If the particles are held in place at this separation distance, they would diffract light.

In further support of this explanation, other investigations using latex with a larger particle size found that the color was red-shifted as the latex diameter was increased. Furthermore, the high $\mathrm{T}_{\mathrm{g}}$ latex became gray with the additions of SWNTs. One reason for this result is that these latex particles are less adhesive and do not cluster around the SWNTs.

\subsection{Film Formation}

Nanocomposite films with good film integrity and noticeable tack were obtained from the low- $\mathrm{T}_{\mathrm{g}}$ latex when cast at room temperature. When casting the blend of the high- $\mathrm{T}_{\mathrm{g}}$ acrylic and $\mathrm{COOH}-\mathrm{CNT}$ solution at room temperature, crack-free, films were obtained, whereas ascast films from the parent latex had micro-cracking and were opaque. The quality of the film formation improved with the addition of the $\mathrm{COOH}-\mathrm{CNT}$ because the dimethyl formamide used in the processing has a plasticizing effect on the acrylic. As the PVA-CNTs were waterborne, they had no plasticizing effect. Hence, nanocomposite films of the high- $\mathrm{T}_{\mathrm{g}}$ latex and the PVA-CNTs were heated at $90{ }^{\circ} \mathrm{C}$ for 30 minutes to achieve good film formation.

\subsection{Dispersion of SWNTs}

Percolation theories predict the volume fraction of nanotubes, $\phi_{c}$, above which they can create a percolating network in an isotropic matrix. This theory treats carbon nanotubes as cylindrical rods that do not interact with each other and are randomly arranged in the matrix. It is known from the theory, ${ }^{20}$ as well as from computer simulations, ${ }^{16}$ that $\phi_{\mathrm{c}}$ for carbon 
nanotubes is inversely proportional to the tube's aspect ratio, $a$, defined as the ratio of its length, $L$, to its diameter, $d$. As an example, for a nanotube with $L=2 \mu \mathrm{m}$ and with $d=2 \mathrm{~nm}$, $a=10^{3}$. Then $\phi_{\mathrm{c}}$ is predicted to be less than $10^{-3}$. Because of the high aspect ratio of CNTs, percolation in latex films has been observed elsewhere at concentrations as low as 0.04 wt.\%. ${ }^{18}$

According to AFM analysis, both types of SWNT ranged in length from $L=0.25 \mu \mathrm{m}$ to 2 $\mu \mathrm{m}$, with the majority being less than $1 \mu \mathrm{m}$ in length. The average $L$ of the COOH-SWNTs was greater than that of the PVA-SWNTs. The values of $d$ ranged between 2 and $6 \mathrm{~nm}$ for the COOH-SWNTs and between 2 and $7 \mathrm{~nm}$ for the PVA-SWNTs. The slightly larger $d$ for the PVA-functionalized SWNTs is attributed to a thin layer of PVA on the nanotube surfaces.

The aspect ratios thus spanned the range from about $10^{2}$ to $10^{3}$. In order to achieve the very low $\phi_{\mathrm{c}}$ values that are predicted by theory, the nanotubes must be finely and randomly dispersed in the polymer matrix.

\subsubsection{Nanocomposites of COOH-SWNTs and Latex}

It is apparent from visual observation of the nanocomposite films that the COOH-SWNTs were not uniformly dispersed in the acrylic matrix. Black specks were visible to the naked eye, especially at higher SWNT concentrations. These specks are attributed to large clusters of nanotubes. Figure 2 shows optical micrographs of nanocomposite films containing COOH-SWNTs. In both types of latex, clusters of nanotubes are apparent, but the size and shape of the clusters differ. The clusters tend to be larger and more needle-like in the low $\mathrm{T}_{\mathrm{g}}$ latex. The surface of the high- $\mathrm{T}_{\mathrm{g}}$ film is rougher, which is attributed to poorer levelling in comparison to the low $\mathrm{T}_{\mathrm{g}}$ latex. 
Atomic force microscopy found regions of the surface of the low $\mathrm{T}_{\mathrm{g}}$ latex in which clusters of the COOH-SWNTs had emerged. By comparison, thorough AFM analysis of the high $\mathrm{T}_{\mathrm{g}}$ nanocomposites did not reveal any nanotubes at the film surface. This result indicates that the clusters that are observed optically are embedded in the film below the surface. Figure 3 shows examples of the two types of nanocomposite film.

There was also evidence for clustering of the COOH-SWNTs in the bulk of the high- $\mathrm{T}_{\mathrm{g}}$ films. Cross-sectional SEM analysis found a heterogeneous distribution of nanotubes, with some regions showing clusters (Figure 4) and other areas having no nanotubes. In the image at higher magnification, the diameter of the nanotube structures is seen to be approximately 30 nm. According to this measurement, this structure is likely to be a bundle of CNTs. Although the resolution is poor, there is evidence in the image for individual nanotubes within the bundle structure. Incidentally, this analysis also found that complete particle coalescence had been achieved. The fracture surfaces have a ridge pattern that is typical of glasses, but there is no evidence for individual acrylic particles.

The nanotube cluster formation is attributed to the relatively poor solubility of the $\mathrm{COOH}$ CNTs in the water/DMF solution. Likewise, in the low- $\mathrm{T}_{\mathrm{g}}$ films, the COOH-SWNTs might adsorb at (or segretate at) the water/air interface because of their relative hydrophobicity. The use of COOH-SWNTs imposes a further disadvantage, as they require an organic solvent (DMF) for their dispersion. COOH-SWNTs are therefore unacceptable for applications that must be waterborne. 


\subsubsection{Nanocomposites of PVA-SWNTs and Latex}

The PVA-SWNTs offer the advantage of completely waterborne processsing. A second important advantage is that the PVA-CNTs are much more finely and uniformly dispersed in the polymer matrix in comparison to the $\mathrm{COOH}-\mathrm{CNTs}$, as found in microscopic analysis.

The good dispersion of the PVA-SWNTs is indicated first of all by their visual appearance. As demonstrated in the photographs in Figure 5, high- $\mathrm{T}_{\mathrm{g}}$ films (35-40 $\mu \mathrm{m}$ thick) are optically transparent up to PVA-SWNT concentrations of about 1 wt.\% (on the polymer). At higher SWNT concentrations, the films develop a gray tint. Films made with the low- $T_{g}$ latex show similar optical transparency at lower PVA-SWNT concentrations. Optical microscopy (Figure 6) of the nanocomposite films found no evidence for nanotube clusters. The microgaphs only show a difference in surface roughness between the low and high- $\mathrm{T}_{\mathrm{g}}$ latex nanocomposites.

AFM analysis of the surfaces of the PVA-SWNT nanocomposites of both types of latex found no evidence for either individual nanotubes or clusters, even at SWNT concentrations as high as $1.4 \mathrm{wt} . \%$ on the polymer. Surveys of the low- $\mathrm{T}_{\mathrm{g}}$ latex surface in SEM analysis revealed a few instances of nanotubes at the air surface. Analysis of the cross-sections of the high- $\mathrm{T}_{\mathrm{g}}$ latex nanocomposites revealed that the nanotubes were uniformly distributed throughout the film. In most cases, the nanotubes are found in isolation rather than in a cluster. Figure 7 gives an example of the observed microstructures. The Au/Pd coating, which was required for SEM analysis, has the effect of masking fine features. It also might make the diameter of the SWNTs appear larger. The resolution of the image is not sufficient to say with certainty whether the SWNTs are exfoliated or are present in the form of ropes or bundles. As there was no plasticizing effect from DMF in this case, the particle coalescence 
is less complete in comparison to the $\mathrm{COOH}-\mathrm{SWNT}$ nanocomposites. Interparticle voids are seen.

\subsection{Optical Absorption of Nanocomposite Films}

Measurements of optical clarity are a good indicator of how finely nanotubes are dispersed in a matrix. Quantitative assessment of the optical clarity of the nanocomposite films is presented in Figure 8. For both types of latex, the optical absorption coefficient of nanocomposite films containing PVA-CNTs is about an order of magnitude lower than that of nanocomposites containing $\mathrm{COOH}-\mathrm{CNT}$. This result is consistent with the microstructural analysis that indicated that the PVA-CNTs are much more finely dispersed in the polymer matrix in comparison to the $\mathrm{COOH}-\mathrm{CNTs}$.

The small amount of PVA that is added to the high- $\mathrm{T}_{\mathrm{g}}$ nanocomposite does not have any noticeable effect on the hydrophilicity of the coating. Water uptake is minimal. The low- $\mathrm{T}_{\mathrm{g}}$ latex exhibits some water-whitening, but this is not adversely affected by the addition of PVA.

\section{Conclusions}

Single-wall carbon nanotubes were functionalized with poly(vinyl alcohol) to render them water-soluble. The CNT solutions were blended with acrylic latex dispersions to produce nanocomposite wateborne coatings. Analysis by microscopy showed that the PVAfunctionalized CNTs were finely dispersed in the polymer matrix. The nanocomposite films exhibited low optical absorption coefficients. It is expected that this type of nanocomposite coating will potentially exhibit high thermal and electrical conductivity along with high wearresistance while offering the advantage of solvent-free processing. 
$\mathrm{COOH}$-functionalized CNTs were dispersed in dimethyl formamide and were similarly blended with latex dispsersions. In contrast to the PVA-functionalized CNTs, this type of nanotube was not as uniformly dispersed in the latex, resulting in higher optical absorption. Microscopy provided evidence for nanotube bundles. In some cases, larger clusters were even apparent to the naked eye.

Further study of the mechanical properties of the nanocomposites is underway in our laboratory. Because the use of surfactants or other dispersants are avoided, the coating's hydrophilicity and barrier properties are expected to be comparable to that of the parent latex coating.

Acknowledgements. P.V.'s stay at the University of Surrey was supported through the SOCRATES student exchange programme. A travel grant from the Royal Society enabled J.L.K. to present this work at the Coatings Science International meeting (COSI 2005) in Noordwijk, The Netherlands. Support for C.L. was provided through the NsHAPe project funded by the European Commission's Framework 6 Programme (Contract NMP3-CT-2004505442).

\section{References}

1. J.M. Salamanca et al., Langmuir 17, (2001), 3202.

2. A.F. Routh, W.B. Zimmerman, Chem. Eng. Sci. 59 (2004) 2961.

3. J.-P. Gorce et al., Eur. Phys. J. E, 8 (2002) 421.

4. A.F. Routh, W.B. Russel, Langmuir, 15 (1999) 7762.

5. A.F. Routh and W.B. Russel, Ind. Eng. Chem. Res. 40 (2001) 4302.

6. D. Juhué and J. Lang, Macromolecules 28 (1995) 1306. 
7. J.L. Keddie, Mater. Sci. Eng. Reports 21 (1997) 101.

8. W.A. de Heer, MRS Bulletin 29 (2004) 281.

9. A.B. Dalton et al., J. Mater. Chem. 14 (2004) 1.

10. R. Andrews et al., Appl. Phys. Lett. 75 (1999) 1329.

11. J.K.W. Sandler et al., Polymer 44 (2003) 5893.

12. J.K.W. Sandler et al., Polymer 45 (2004) 2001.

13. B.P. Grady et al., J. Phys. Chem. B, 106 (2002) 5852.

14. R. Sainz et al., Nanotechnology, 16 (2005) S150.

15. Y. Lin et al., Macromolecules, 36 (2003) 7199.

16. M. Foygel et al., Phys. Rev. B, 71 (2005) 104201.

17. A. Dufresne et al., J. Mat. Sci., 37 (2002) 3915.

18. O. Regev et al., Adv. Mater., 16 (2004) 248.

19. J.C. Grunlan et al., Adv. Mater., 16 (2004) 150.

20. I. Balberg et al., Phys. Rev. B, 30 (1984) 3933. 


\section{Figure Captions}

Figure 1. AFM images (height image on the left and phase contrast image on the right) of a dilute blend of the low- $\mathrm{T}_{\mathrm{g}}$ latex and $\mathrm{COOH}-\mathrm{CNTs}(0.4 \mathrm{wt} \%)$ cast on a mica surface. The arrows indicate where three latex particles are clustered on a SWNT. Area of image is $6 \mu \mathrm{m} x$ $6 \mu \mathrm{m}$.

Figure 2. Optical micrographs of nanocomposite films containing COOH-SWNTs. (a) 0.2 wt.\% COOH-SWNTs in the high- $\mathrm{T}_{\mathrm{g}}$ latex. (b) $0.15 \mathrm{wt} \% \mathrm{COOH}-\mathrm{SWNT}$ in the low- $\mathrm{T}_{\mathrm{g}}$ latex. The scale bar represents $200 \mu \mathrm{m}$.

Figure 3. AFM images of the surfaces of latex films containing COOH-SWNTs. (a) Phase image of the low-Tg latex with 0.4 wt.\% COOH-SWNTs. (b) Topographic image of the high-Tg latex with 0.2 wt.\% COOH-SWNTs. Each image shows a $10 \mu \mathrm{m}$ x $10 \mu \mathrm{m}$ area. Figure 4. SEM image of the cross-section of a nanocomposite film of the high- $\mathrm{T}_{\mathrm{g}}$ latex and 0.27 wt.\% COOH-SWNTs. (a) Lower magnification (b) Higher magnification.

Figure 5. Image of nanocomposite films made from PVA-SWNTs and the high- $\mathrm{T}_{\mathrm{g}}$ acrylic latex and cast on glass plates. The SWNT concentration (wt.\% on the polymer) is indicated. Figure 6. Optical micrographs of nanocomposite films containing PVA-SWNTs. (a) 2.5 wt.\% PVA-SWNTs in the high-T $\mathrm{T}_{\mathrm{g}}$ latex. (b) $3.1 \mathrm{wt} . \%$ PVA-SWNTs in the low- $\mathrm{T}_{\mathrm{g}}$ latex. The scale bar represents $200 \mu \mathrm{m}$.

Figure 7. SEM image of the cross-section of a nanocomposite film of the high- $\mathrm{T}_{\mathrm{g}}$ latex and 1.26 wt.\% PVA-CNTs. (a) Lower magnification (b) Higher magnification.

Figure 8. Optical absorption coefficients at a wavelength of $600 \mathrm{~nm}$ for four types of nanocomposite film. Triangles indicate nanocomposites containing COOH-CNTs; squares indicate PVA-CNTs. The filled symbols are for the low- $\mathrm{T}_{\mathrm{g}}$ latex; the open symbols are for the high- $\mathrm{T}_{\mathrm{g}}$ latex. 


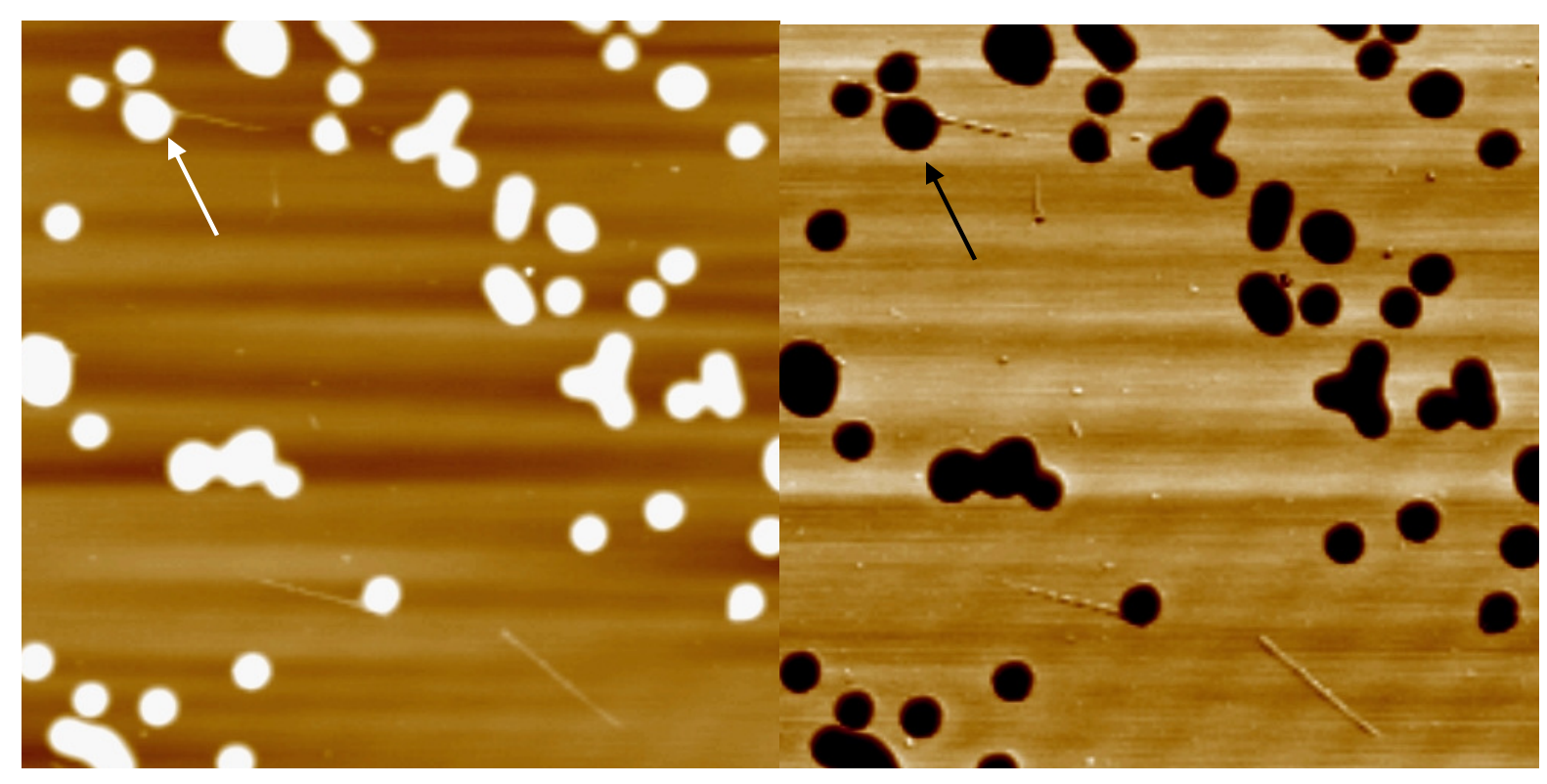

Figure 1. AFM images (height image on the left and phase contrast image on the right) of a dilute blend of the low- $\mathrm{T}_{\mathrm{g}}$ latex and $\mathrm{COOH}-\mathrm{CNTs}(0.4 \mathrm{wt} \%)$ cast on a mica surface. The arrows indicate where three latex particles are clustered on a SWNT. Area of image is $6 \mu \mathrm{m} x$ $6 \mu \mathrm{m}$. 

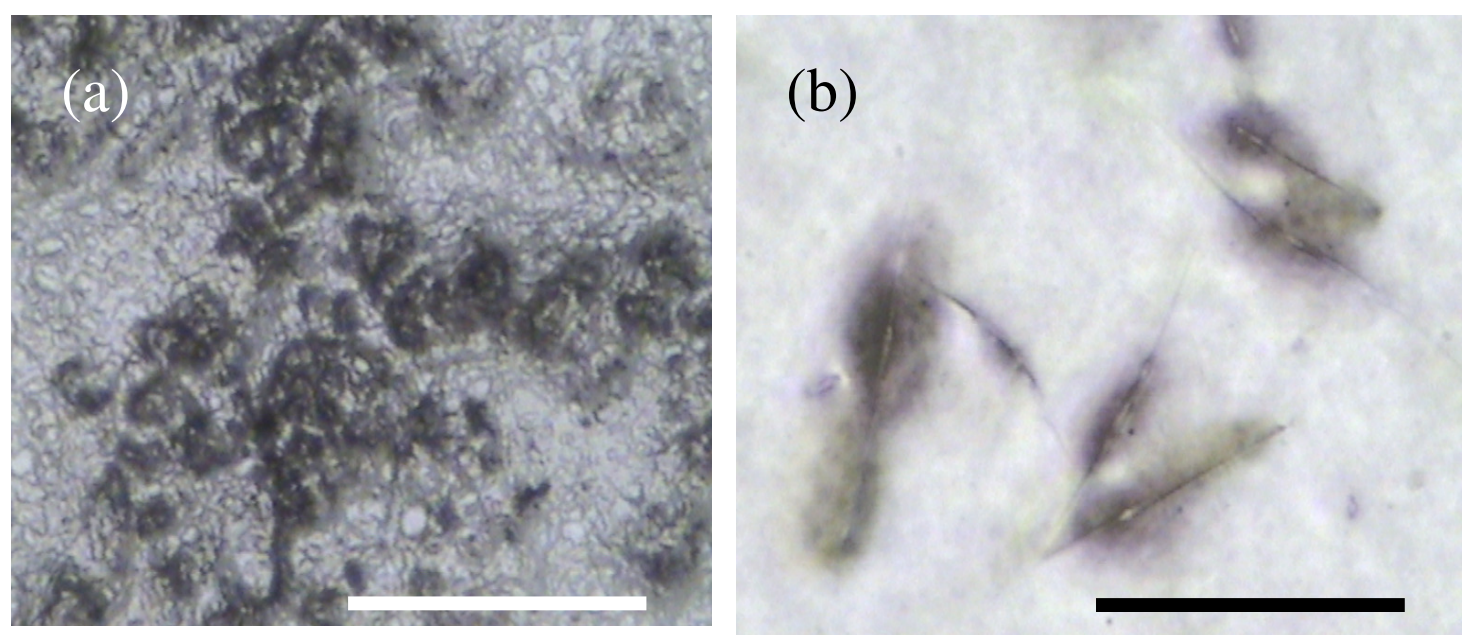

Figure 2. Optical micrographs of nanocomposite films containing COOH-SWNTs. (a) 0.2 wt. $\%$ COOH-SWNTs in the high- $\mathrm{T}_{\mathrm{g}}$ latex. (b) $0.15 \mathrm{wt} \% \mathrm{COOH}-\mathrm{SWNT}$ in the low- $\mathrm{T}_{\mathrm{g}}$ latex. The scale bar represents $200 \mu \mathrm{m}$. 

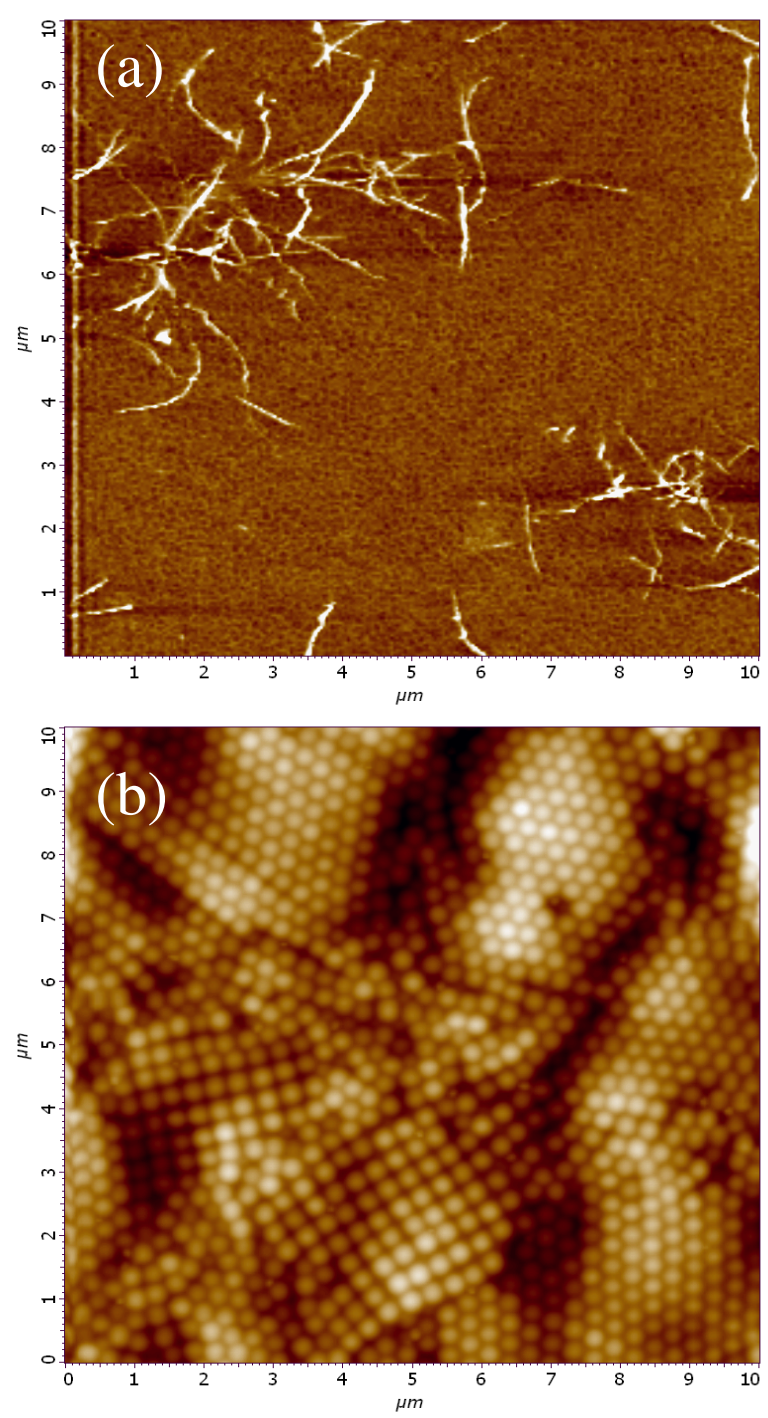

Figure 3. AFM images of the surfaces of latex films containing COOH-SWNTs. (a) Phase image of the low-Tg latex with 0.4 wt.\% COOH-SWNTs. (b) Topographic image of the high-Tg latex with $0.2 \mathrm{wt} \% \mathrm{COOH}-\mathrm{SWNTs}$. Each image shows a $10 \mu \mathrm{m}$ x $10 \mu \mathrm{m}$ area. 
Published in Progress in Organic Coatings (2006) 57(2), 91-97
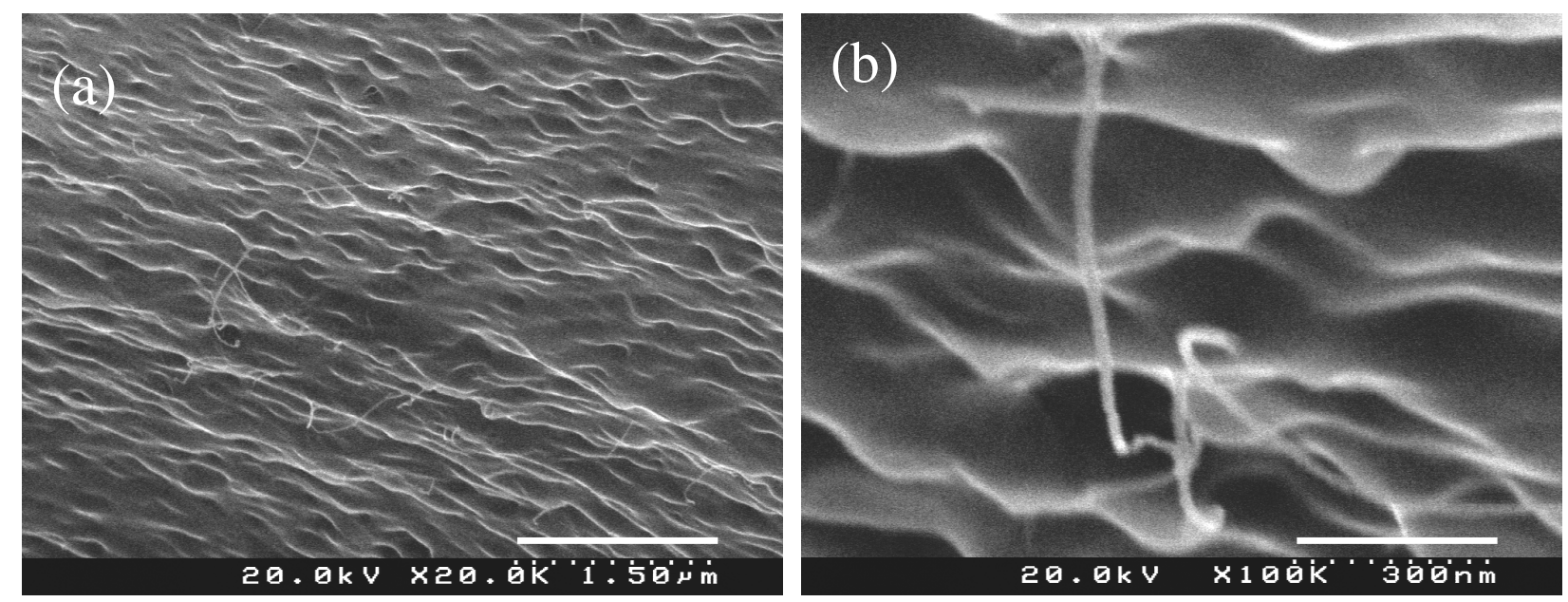

Figure 4. SEM image of the cross-section of a nanocomposite film of the high- $\mathrm{T}_{\mathrm{g}}$ latex and 0.27 wt.\% COOH-SWNTs. (a) Lower magnification (b) Higher magnification. 


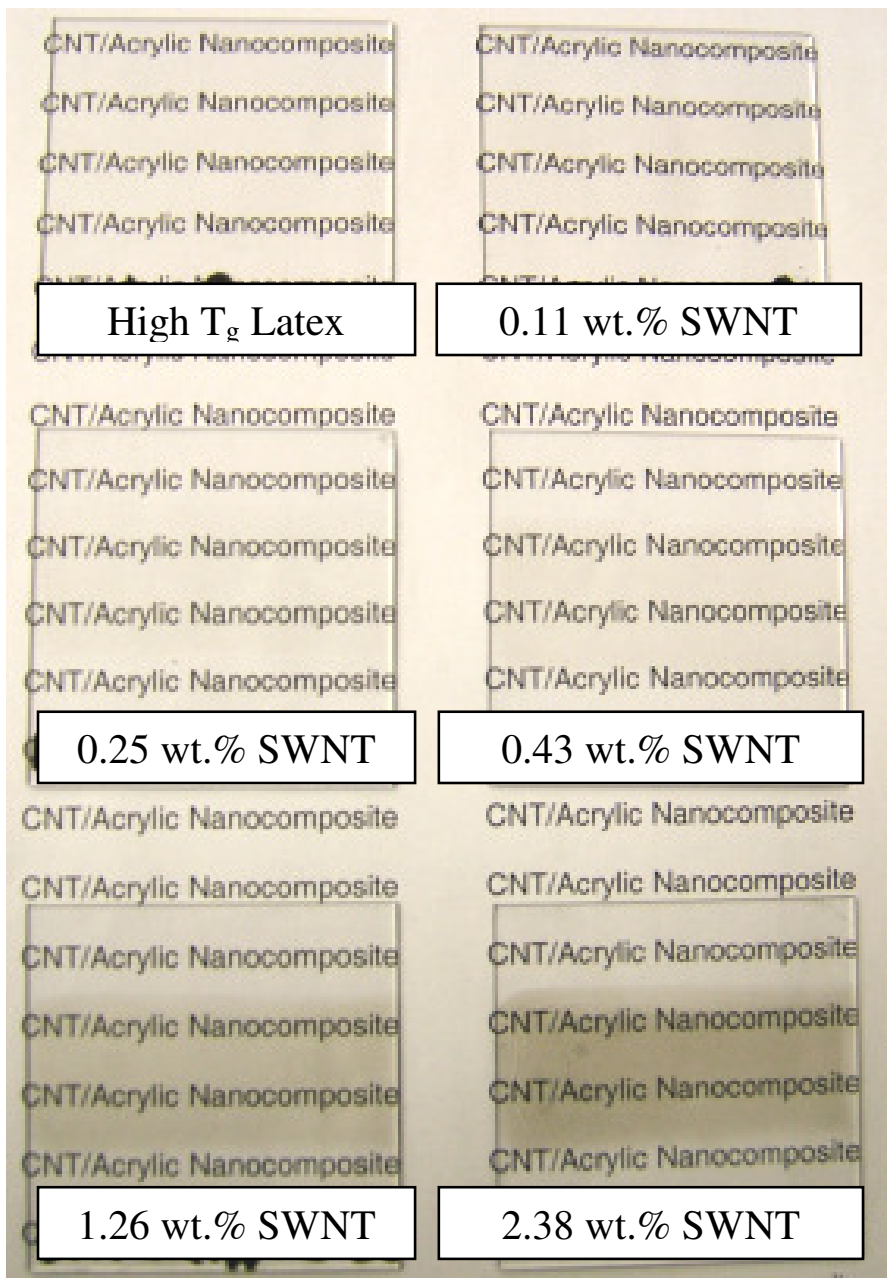

Figure 5. Image of nanocomposite films made from PVA-SWNTs and the high- $\mathrm{T}_{\mathrm{g}}$ acrylic latex and cast on glass plates. The SWNT concentration (wt.\% on the polymer) is indicated. 
Published in Progress in Organic Coatings (2006) 57(2), 91-97
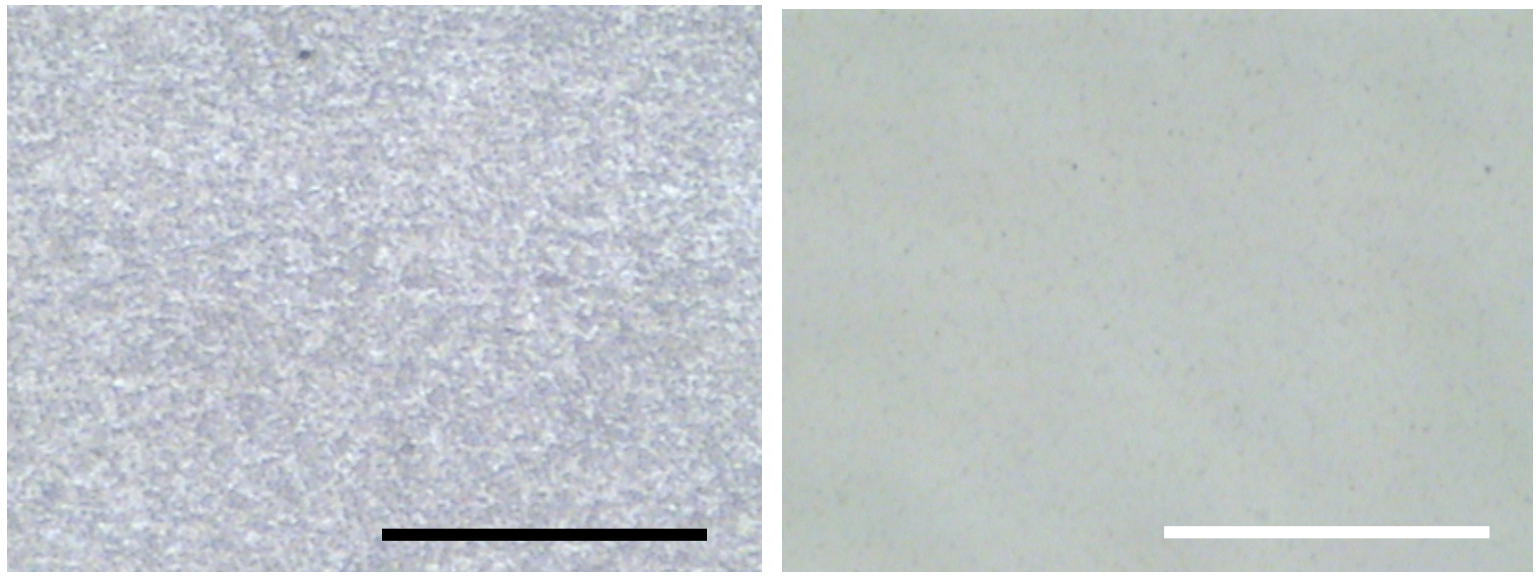

Figure 6. Optical micrographs of nanocomposite films containing PVA-SWNTs. (a) 2.5

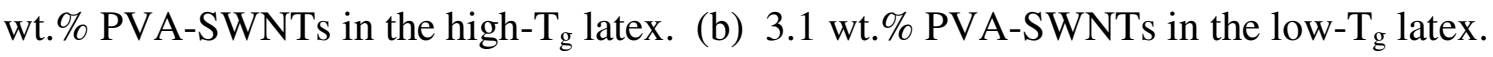
The scale bar represents $200 \mu \mathrm{m}$. 

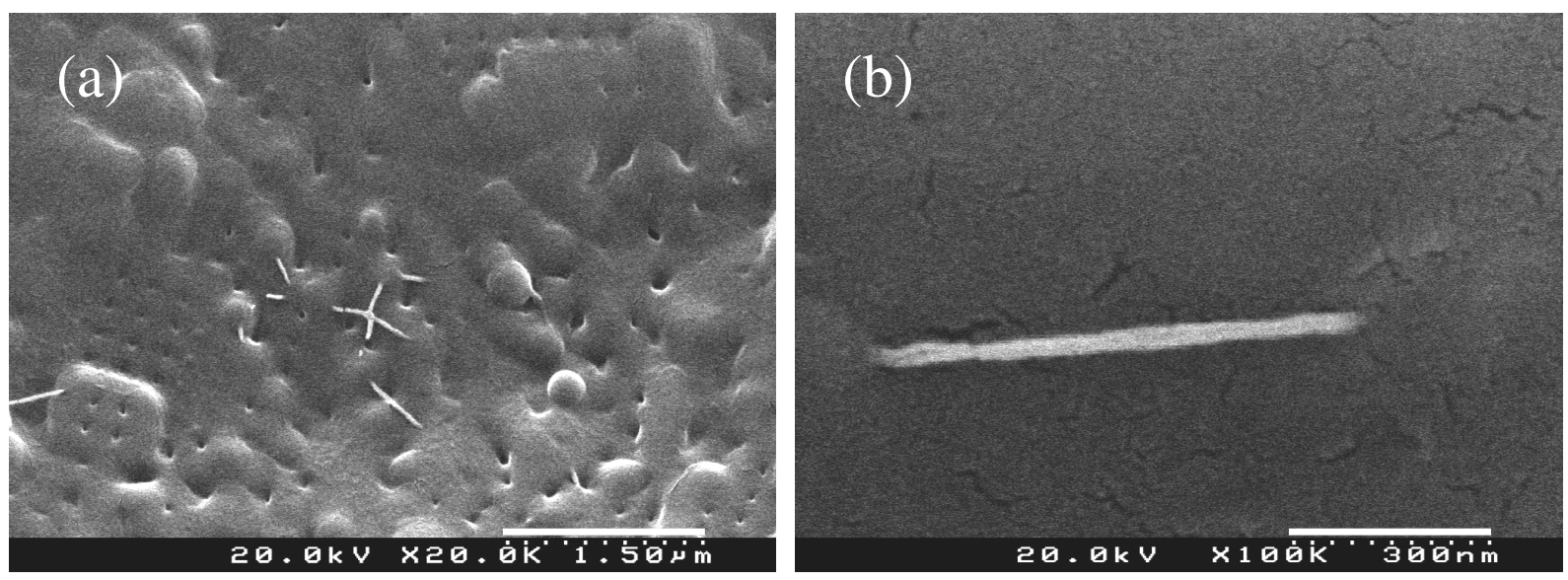

Figure 7. SEM image of the cross-section of a nanocomposite film of the high- $\mathrm{T}_{\mathrm{g}}$ latex and 1.26 wt.\% PVA-CNTs. (a) Lower magnification (b) Higher magnification. The cracks that appear in (b) are in the Au coating. 
Published in Progress in Organic Coatings (2006) 57(2), 91-97

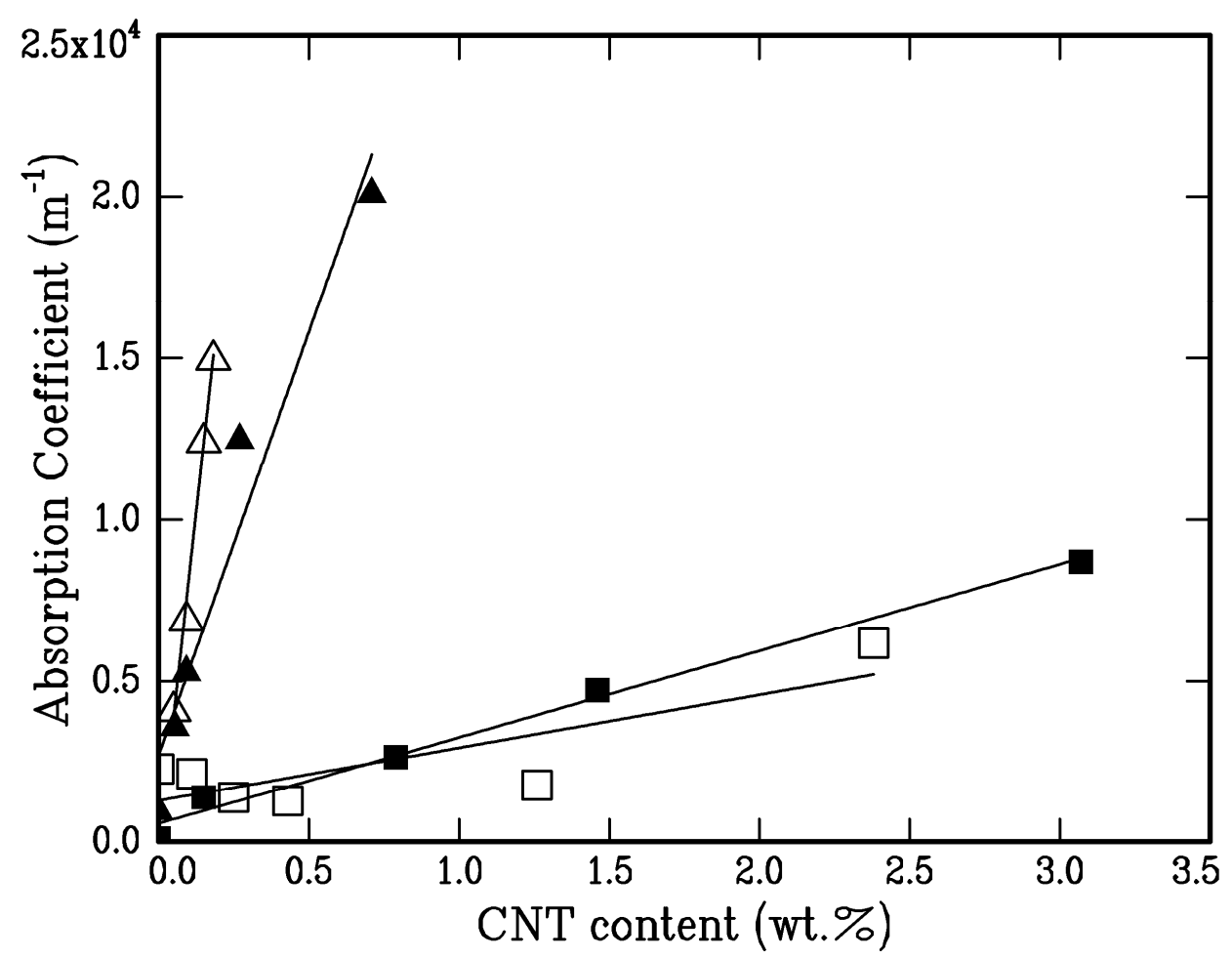

Figure 8. Optical absorption coefficients at a wavelength of $600 \mathrm{~nm}$ for four types of nanocomposite film. Triangles indicate nanocomposites containing COOH-CNTs; squares indicate those with PVA-CNTs. The filled symbols are for the low- $\mathrm{T}_{\mathrm{g}}$ latex; the open symbols are for the high- $\mathrm{T}_{\mathrm{g}}$ latex. 